\title{
Transmit Beampattern Synthesis with Constant Beamwidth and Sidelobe Control for Wideband MIMO Radar
}

\author{
Pengcheng Gong and Zhenhai Shao \\ Greating-UESTC Joint Experiment Engineering Center, School of Communication and Information Engineering, \\ University of Electronic Science and Technology of China, Chengdu 611731, China
}

Correspondence should be addressed to Zhenhai Shao; shao_zh@uestc.edu.cn

Received 7 January 2014; Revised 10 May 2014; Accepted 13 May 2014; Published 22 June 2014

Academic Editor: Shengqi Zhu

Copyright (C) 2014 P. Gong and Z. Shao. This is an open access article distributed under the Creative Commons Attribution License, which permits unrestricted use, distribution, and reproduction in any medium, provided the original work is properly cited.

\begin{abstract}
A beampattern synthesis approach is proposed to design the power spectral density matrix (PSDM), which is chosen to achieve a given transmit beampattern in wideband multiple-input multiple-output (MIMO) radar systems. The proposed approach focuses on transmit beampattern synthesis with constant beamwidth and sidelobe control. Moreover, the design problem is further converted to a convex optimization problem, which is solved efficiently via the modeling system CVX. In comparison to these recently developed wideband MIMO beampattern synthesis methods, the proposed approach maintains a constant beamwidth across the entire frequency band and provides a great improvement in sidelobe control. Numerical simulation results are obtained to validate the effectiveness of this approach.
\end{abstract}

\section{Introduction}

Transmit beampattern synthesis is a well-studied topic in standard phased-array radars [1-3]. Recently, several transmit beampattern design approaches for narrowband MIMO radars have been reported [4-9]. The main idea of these approaches is to design the proper probing signals according to the desired transmit beampattern. A relationship between the spatial beampattern and the covariance matrix of the transmit waveforms derived in $[4,5]$ shows that it is possible to design a covariance matrix to synthesize the desired beampattern. This work is further extended in $[6,7]$ to solve the beampattern design problem by a semidefinite quadratic programming (SQP) algorithm.

However, the discussion is limited to transmit beampattern synthesis for wideband MIMO radar. Antonio and Fuhrmann have designed the signal cross-spectral density matrixes (CSDM) in order to approximate the desired transmit beampattern in [10]. He et al. [11] also have proposed a wideband beampattern formation via iterative techniques (WBFIT) to synthesize wideband MIMO waveforms, which satisfy some practical constraints such as constant-modulus or low peak-to-average power ratio (PAPR). In WBFIT, the beamwidth of the designed beampattern tends to shrink as the frequency increases for one narrow mainbeam. The method in [12] performs similarly as WBFIT, but with much lower computational costs.

Although these methods [10-12] have discussed the wideband beampattern synthesis problem, so little attention has been paid to the case of the constant beamwidth and the sidelobe attenuation. In this paper, we propose a beampattern synthesis approach to design power spectral density matrix (PSDM) in order to match a transmit energy distribution in both space and frequency for wideband MIMO radar. In our proposed approach, the mainlobe of the designed beampattern has an almost constant beamwidth for different frequencies and the sidelobe level of the designed beampattern is strictly below a desired sidelobe value. In addition, the proposed approach is converted to second-order cone programming (SOCP) problem, which is easily solved by the convex optimization toolbox CVX. Simulation results show that the proposed approach significantly outperforms the existing WBFIT approach in terms of both frequency invariant property and sidelobe attenuation.

The rest of this paper is organized as follows. The problem is formulated in Section 2. In Section 3, the proposed 
approach is developed. Design examples and simulation results are shown in Section 4 and concluded remarks are given in Section 5.

Notation 1. We denote vectors and matrices by lowercased and uppercased letters, respectively. $(\cdot)^{H}$ denotes the vector or matrix conjugate transpose operation, $(\cdot)^{T}$ denotes the transpose operation, $(\cdot)^{*}$ denotes the conjugate operation, $\operatorname{diag}(\cdot)$ denotes diagonals of a matrix, $|\cdot|$ denotes the absolute value operation, $\|\cdot\|_{2}$ denotes the Euclidean norm, and $\operatorname{rank}(\cdot)$ refers to the rank operation.

\section{Problem Formulation}

When a MIMO radar system employs $M$ transmit antennas with the interelement spacing $d$, the signal transmitted by the $m$ th antenna is given by

$$
s_{m}(t)=x_{m}(t) \cdot e^{j 2 \pi f_{c} t}
$$

where $f_{c}$ denotes the carrier frequency of the transmitted signal and $x_{m}(t)$ is the baseband signal, whose time support is $[0, \tau]$.

In this case, the far-field signal at target angle $\theta$ can be written as

$$
z_{\theta}(t)=\sum_{m=0}^{M-1} s_{m}\left(t-\tau_{m}\right), \quad t \in[0, \tau],
$$

where $\tau_{m}$ is the time needed by the signal emitted from the $m$ th transmit antenna to the target. Substituting (1) into (2), (2) is then modified as

$$
z_{\theta}(t)=\sum_{m=0}^{M-1} x_{m}\left(t-\tau_{m}\right) \cdot e^{j 2 \pi f_{c}\left(t-\tau_{m}\right)}, \quad t \in[0, \tau] .
$$

Assuming that the Fourier transform (FT) of $x_{m}(t)$ is $y_{m}(f),(3)$ can be rewritten as

$$
z_{\theta}(t)=\int_{-B / 2}^{B / 2} Y(\theta, f) e^{j 2 \pi\left(f+f_{c}\right) t} d f
$$

where

$$
Y(\theta, f)=\sum_{m=0}^{M-1} y_{m}(f) \cdot e^{-j 2 \pi\left(f+f_{c}\right) \tau_{m}}=a^{H}(\theta, f) \cdot y(f),
$$

$a(\theta, f)=\left[1, e^{j 2 \pi\left(f+f_{c}\right) \tau_{0}}, \ldots, e^{j 2 \pi\left(f+f_{c}\right) \tau_{m}}, \ldots, e^{j 2 \pi\left(f+f_{c}\right) \tau_{M-1}}\right]^{T}$,

$$
y(f)=\left[y_{0}(f), y_{1}(f), \ldots, y_{m}(f), \ldots, y_{M-1}(f)\right]^{T} .
$$

Enlightened by these approaches proposed in $[10,11]$, the beampattern at spatial angle $\theta$ and frequency $f+f_{c}$ is redefined as (see [13] for details)

$$
P\left(\theta, f+f_{c}\right)=a^{H}(\theta, f) \cdot y(f) \cdot y^{H}(f) \cdot a(\theta, f),
$$

where the baseband frequency $f \in[-B / 2, B / 2]$ and the spatial angle $\theta \in[0, \pi]$. In the case when wideband signals are used, the PSDM is defined as [14]

$$
S(f)=y(f) \cdot y^{H}(f) .
$$

Then (8) can be rewritten as

$$
P\left(\theta, f+f_{c}\right)=a^{H}(\theta, f) \cdot S(f) \cdot a(\theta, f) .
$$

In practical applications, the baseband signal and the spatial angle need to be discretized, so that (6) and (7) can be rewritten, respectively, as follows:

$$
\begin{gathered}
a_{k p}=a\left(\theta_{k}, \frac{p}{N T_{s}}\right) \\
=\left[1, e^{j 2 \pi\left(f+p / N T_{s}\right) \tau_{0}}, \ldots, e^{j 2 \pi\left(f+p / N T_{s}\right) \tau_{m}},\right. \\
\left.\ldots, e^{j 2 \pi\left(f+p / N T_{s}\right) \tau_{M-1}}\right]^{T}, \\
y_{p}=\left[y_{0}(p), y_{1}(p), \ldots, y_{m}(p), \ldots, y_{M-1}(p)\right]^{T},
\end{gathered}
$$

where $\left\{\theta_{k}\right\}_{k=1}^{K}$ is a fine grid of points that cover the spatial angle interval $[0, \pi]$. Note that $\left\{y_{p}\right\}_{p=-N / 2}^{N / 2-1}$ represents the discrete Fourier transform (DFT) of

$$
\begin{array}{r}
x(n)=\left[x_{0}(n), \ldots, x_{m}(n), \ldots, x_{M-1}(n)\right]^{T}, \\
n=0, \ldots, N-1,
\end{array}
$$

where $N$ denotes the number of samples and is assumed to be even.

So, the beampattern in (8) can be expressed on the discrete angle-frequency grid as

$$
P_{k p}=a_{k p}^{H} S(p) a_{k p}
$$

where $k \in[1, K], p \in[-N / 2, \ldots, 0, \ldots, N / 2-1]$,

$$
S(p)=y_{p} y_{p}^{H}
$$

In this paper, when the transmit beampattern and a desired beampattern are defined as (13) and $d_{k p}$, respectively, the goal is to choose the appropriate PSDM $S(p)(p \in$ $[-N / 2, \ldots, 0, \ldots, N / 2-1])$, under some constraints, so that the beampattern in (13) matches the desired one $d_{k p}$.

\section{The Proposed Transmit Beampattern Design}

In this section, how to synthesize wideband transmit beampattern with constant beamwidth across the entire frequency band and sidelobe control is described. Here, the beampattern synthesis goal is to minimize the error between the designed beampattern and the desired one over the mainlobe region and to maintain the sidelobe region to be lower than 
a given threshold value. Mathematically, such beampattern synthesis problem can be formulated as follows:

$$
\begin{gathered}
\min _{\{S(p)\}}\left\{\sum_{m^{\prime}=1}^{M^{\prime}} \sum_{p=-N / 2}^{N / 2-1}\left|\alpha d_{m^{\prime} p}-a_{m^{\prime} p}^{H} S(p) a_{m^{\prime} p}\right|^{2}\right\} \\
\theta_{m^{\prime}} \in \Theta_{M^{\prime} L}, \quad m^{\prime}=1, \ldots, M^{\prime} \\
\text { subject to }\left|a_{s p}^{H} S(p) a_{s p}\right| \leq \sigma, \quad \theta_{s} \in \Theta_{S L}, s=1, \ldots, S,
\end{gathered}
$$

where $\theta_{m^{\prime}} \in \Theta_{M^{\prime} L}\left(m^{\prime}=1, \ldots, M^{\prime}\right)$ and $\theta_{s} \in \Theta_{S L}(s=$ $1, \ldots, S)$ that denote that a fine grid (uniform or nonuniform) of points covers mainlobe region and sidelobe region of interest, respectively. $d_{m^{\prime} p}$ and $\sigma$ denote the mainlobe region and the desired sidelobe value of a desired beampattern, respectively. And $\alpha>0$ is a variable to control the magnitude of the desired beampattern.

In (15), the PSDM cannot be chosen freely and should satisfy the following constraints:

$$
\begin{gathered}
C_{1}: \sum_{n=0}^{N-1}\left|x_{m}(n)\right|^{2}=\frac{c}{M}, \quad m=0, \ldots, M-1 ; \\
C_{2}: S(p) \geq 0, \quad p \in\left[-\frac{N}{2}, \frac{N}{2}-1\right],
\end{gathered}
$$

where $c / M$ is the transmitted power from each antenna, and $c$ is a total transmit power for $M$ antennas. The first constraint $C_{1}$ means that transmit power from all the antenna elements should be the same, and the second constraint $C_{2}$ ensures that $S(p)$ is a positive semidefinite matrix.

Using the Parseval equality, the first constraint $C_{1}$ on $\left\{x_{m}(n)\right\}$ imposes the following constraint on $\left\{y_{m}(p)\right\}$ :

$$
\begin{array}{r}
\sum_{p=-N / 2}^{N / 2-1}\left|y_{m}(p)\right|^{2}=N \sum_{n=0}^{N-1}\left|x_{m}(n)\right|^{2}=\frac{N c}{M} \\
m=0, \ldots, M-1 .
\end{array}
$$

Therefore, $C_{1}$ in (16a) can be rewritten as

$$
\sum_{p=-N / 2}^{N / 2-1} S_{m}(p)=\frac{N c}{M}, \quad m=0, \ldots, M-1
$$

Combining (15) with the above PSDM constraints in (16a) and (16b), the beampattern design problem can be formulated as

$$
\begin{array}{r}
\min _{\{S(p)\}}\left\{\sum_{m^{\prime}=1}^{M^{\prime}} \sum_{p=-N / 2}^{N / 2-1}\left|\alpha d_{m^{\prime} p}-a_{m^{\prime} p}^{H} S(p) a_{m^{\prime} p}\right|^{2}\right\} \\
\theta_{m^{\prime}} \in \Theta_{M^{\prime} L}, \quad m^{\prime}=1, \ldots, M^{\prime}
\end{array}
$$

subject to $\quad\left|a_{s p}^{H} S(p) a_{s p}\right| \leq \sigma \quad \theta_{s} \in \Theta_{S L}, s=1, \ldots, S$

$$
\begin{gathered}
\sum_{p=-N / 2}^{N / 2-1} S_{m}(p)=\frac{N c}{M}, \quad m=0, \ldots, M-1 \\
S(p) \geq 0, \quad p \in\left[-\frac{N}{2}, \frac{N}{2}-1\right] .
\end{gathered}
$$

Next, it will be shown that the optimal design problem (19) can be converted to a second-order cone programming (SOCP) [15] and can be efficiently solved by the wellestablished interior point method [16], such as public domain software CVX toolbox [17].

Introducing new variables $\tau_{p}(p \in[-N / 2, N / 2-1])$, (19) can be converted to the following form:

$$
\begin{gathered}
\min _{\{S(p)\}} \sum_{p=-N / 2}^{N / 2-1} \tau_{p} \\
\text { subject to }\left\{\sum_{m^{\prime}=1}^{M^{\prime}}\left|\alpha d_{m^{\prime} p}-a_{m^{\prime} p}^{H} S(p) a_{m^{\prime} p}\right|^{2}\right\} \leq \tau_{p}, \\
\qquad a_{s p}^{H} S(p) a_{s p} \mid \leq \sigma, \quad \theta_{m^{\prime}} \in \Theta_{M^{\prime} L}, \quad \Theta_{S L}^{\prime}, s=1, \ldots, M^{\prime} \\
\sum_{p=-N / 2}^{N / 2-1} S_{m}(p)=\frac{N c}{M}, \quad m=0, \ldots, M-1 \\
\quad S(p) \geq 0, \quad p \in\left[-\frac{N}{2}, \frac{N}{2}-1\right] .
\end{gathered}
$$

The first constraint and the second constraint in (20) can be rewritten as the following compact form, respectively:

$$
\begin{gathered}
\left\|\alpha d_{p}-\operatorname{diag}\left(A_{p} S(p) A_{p}^{H}\right)\right\| \leq \sqrt{\tau_{p}} \\
\left|\operatorname{diag}\left(\bar{A}_{p} S(p) \bar{A}_{p}^{H}\right)\right| \leq \sigma,
\end{gathered}
$$

where $A_{p}=\left[a_{1 p}^{*}, \ldots, a_{m^{\prime} p}^{*}, \ldots, a_{M^{\prime} p}^{*}\right]^{T}$ is the steering matrix of the mainlobe region, $\bar{A}_{p}=\left[a_{1 p}^{*}, \ldots, a_{s p}^{*}, \ldots, a_{S p}^{*}\right]^{T}$ is the steering matrix of the sidelobe region, and $d_{p}=\left[d_{1 p}, \ldots\right.$, $\left.d_{m^{\prime} p}, \ldots, d_{M^{\prime} p}\right]^{T}$.

Based on (21), the beampattern design in (19) can be expressed as the following minimization problem:

$$
\min _{\{S(p)\}} \sum_{p=-N / 2}^{N / 2-1} \tau_{p}
$$

subject to $\left\|\alpha d_{p}-\operatorname{diag}\left(A_{p} S(p) A_{p}^{H}\right)\right\| \leq \sqrt{\tau_{p}}$

$$
\begin{gathered}
\left|\operatorname{diag}\left(\bar{A}_{p} S(p) \bar{A}_{p}^{H}\right)\right| \leq \sigma \\
\sum_{p=-N / 2}^{N / 2-1} S_{m}(p)=\frac{N c}{M}, \quad m=0, \ldots, M-1 \\
S(p) \geq 0, \quad p \in\left[-\frac{N}{2}, \frac{N}{2}-1\right] .
\end{gathered}
$$




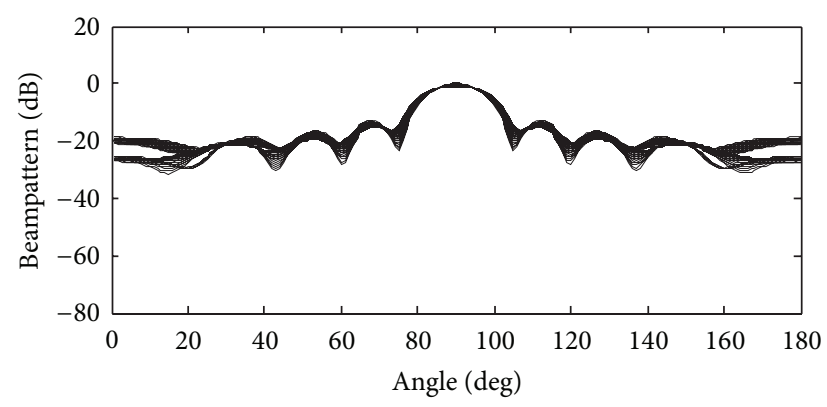

- The WBFIT approach

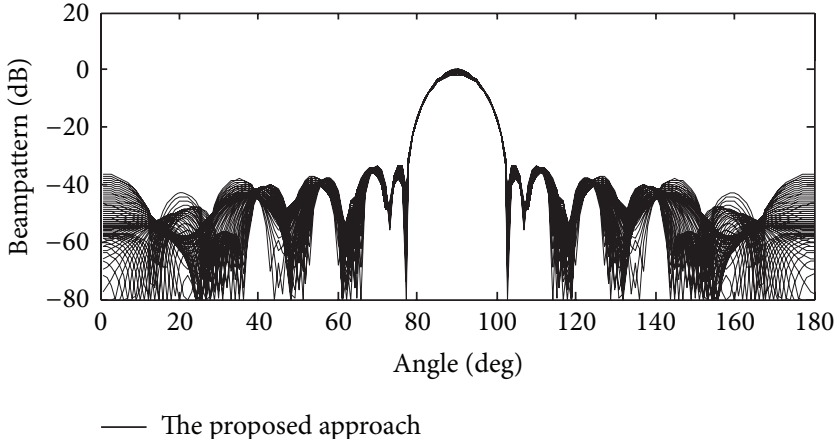

(a)

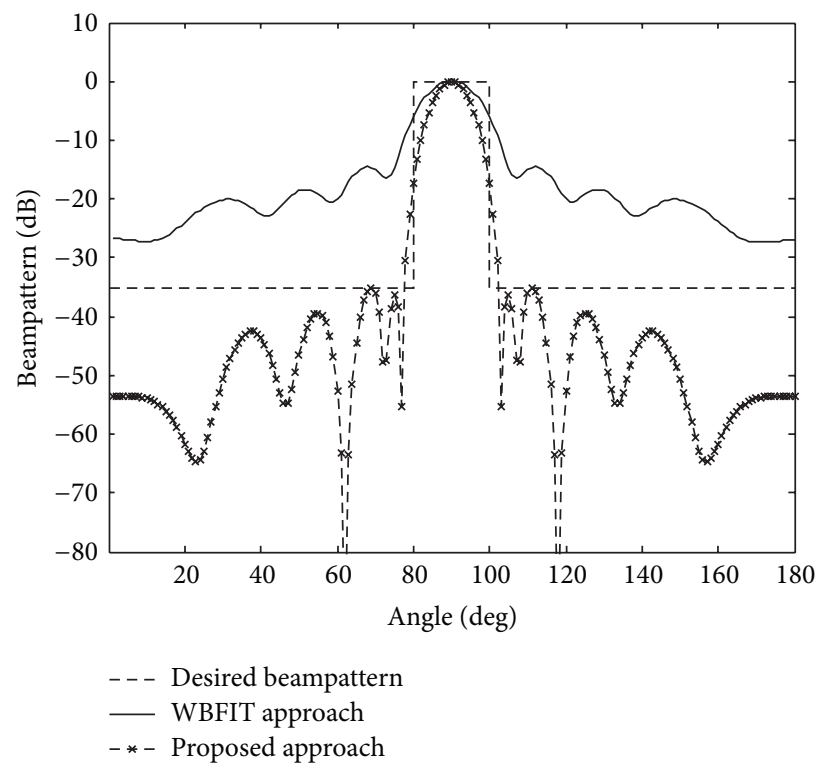

(b)

FIGURE 1: Comparison of the synthesized beampattern. The desired beampattern is given in (23). (a) Plot at all the 64 frequencies using the WBFIT approach and the proposed approach. (b) Plot at center frequency.

It can be observed that, by introducing new variables, the optimal design problem in (19) can be converted to the SOCP problem (22), which is a convex optimization problem [15]. The second-order cone, $Q^{m}=\left\{(x, y) \in R^{m} \times R \mid\|x\|_{2} \leq\right.$ $y$, can be solved using CVX in MATLAB with the code given in " $\{x, y\}\langle\operatorname{In}\rangle$ complex_lorentz (m)." We therefore emphasize more on convex problem formulation (22) and the computational complexity analysis for the solution is not provided.

Now, if $S(p)$ is of rank one, an optimal solution to (14) can be obtained. However, if the corresponding rank is greater than one, we need to resort to randomization techniques to extract a feasible solution [18].

Let $S(p)=\sum_{i=1}^{r} \lambda_{i} q_{i} q_{i}^{T}$ denote the eigenvalue decomposition of $S(p)$, where $r=\operatorname{rank}[S(p)]$, the eigenvalues are $\lambda_{1} \geq \lambda_{2} \geq \cdots \geq \lambda_{r}>0$, and $q_{1}, \ldots, q_{r}$ are the respective eigenvectors. We choose $y_{p}=\sqrt{\lambda}_{1} q_{1}$ as our candidate vector to $(14)$.
Hence the proposed approach works as follows. Firstly, the frequency band occupied by the wideband signal is divided into a certain number of narrowband bins. Secondly, PSDM of the signal vectors at each frequency bin is designed via proposed approach in (22). Then, a feasible solution to (14) from a solution $S(p)$ is extracted.

\section{Numerical Results}

This section focuses on demonstrating the performances of the proposed approach using an $M=10$ MIMO radar system with uniform linear array (ULA), where the interelement spacing $d$ is given by half-wavelength. The parameters for simulations are set as follows: $f_{c}=1 \mathrm{GHz}, B=200 \mathrm{MHz}$, $N=64, K=180$, and the total transmit power is $c=1$. Although the design of multiple beams is not considered in these simulations, it can be achieved via the proposed 


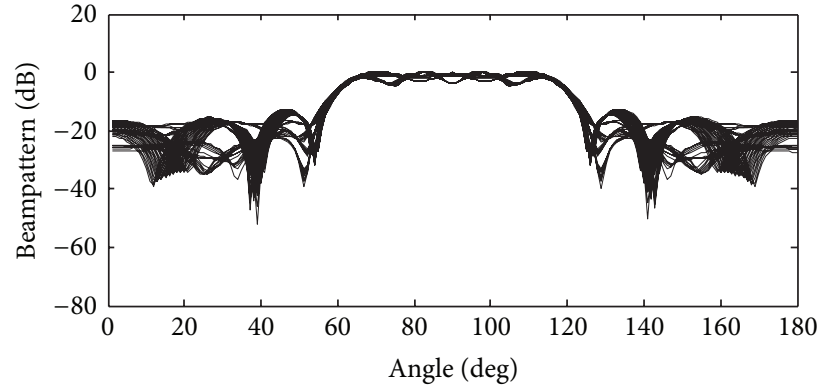

— The WBFIT approach

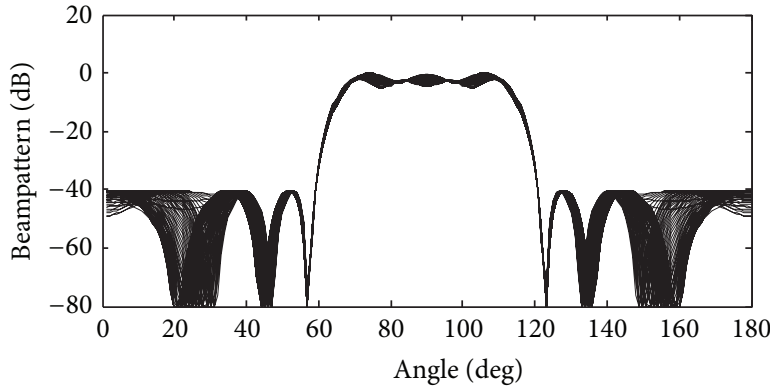

— The proposed approach

(a)

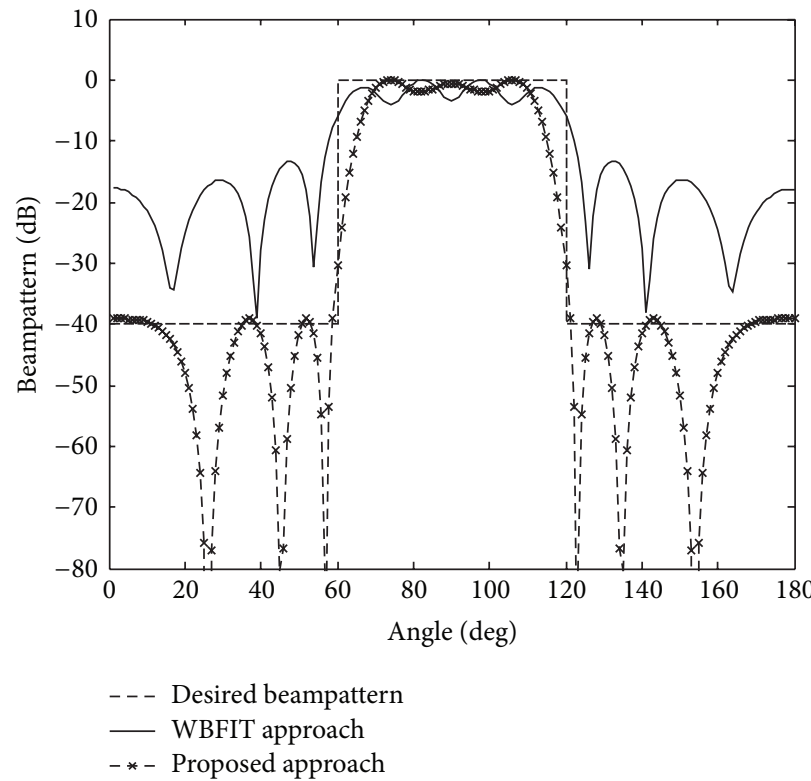

(b)

Figure 2: Comparison of the synthesized beampattern. The desired beampattern is given in (24). (a) Plot using the WBFIT approach and the proposed approach. (b) Plot at center frequency.

approach. So, it is sufficient only to discuss the single beam design.

Firstly, the desired beampattern which is synthesized using the proposed approach denotes the following:

$$
\begin{gathered}
d(\theta, f)= \begin{cases}1, & 80^{\circ} \leq \theta_{k} \leq 100^{\circ} \\
0, & \text { others, }\end{cases} \\
f \in\left[-\frac{B}{2}+f_{c}, \frac{B}{2}+f_{c}\right] .
\end{gathered}
$$

Here the mainlobe region is set to $\Theta_{M^{\prime} L}=\left[80^{\circ}, 100^{\circ}\right]$; that is, the beamwidth of the desired beampattern is $20^{\circ}$, and the sidelobe region is $\Theta_{S L}=\left[1^{\circ}, 79^{\circ}\right] \cup\left[101^{\circ}, 180^{\circ}\right]$, with $1^{\circ}$ increment between adjacent grid points.

Figure 1(a) shows the obtained beampatterns at all the 64 frequencies using the WBFIT approach of [11] and the proposed approach in (19). In this case, the desired sidelobe value is set to $\sigma=-35 \mathrm{~dB}$. From the plots, it can been seen that the beampattern obtained by proposed approach shows a clean mainlobe at $90^{\circ}$ across the entire frequency range, and the main beamwidth synthesized by proposed approach keeps fixed for all in-band frequency. Figure 1(b) compares the beampattern designed by the proposed approach with the WBFIT approach at center frequency. From Figure 1(b), it is noted that the designed beampattern using the proposed approach has a sidelobe level of $-35 \mathrm{~dB}$.

Secondly, the following desired beampattern with a wider mainlobe is considered:

$$
\begin{gathered}
d(\theta, f)= \begin{cases}1, & 60^{\circ} \leq \theta_{k} \leq 120^{\circ} \\
0, & \text { others, }\end{cases} \\
f \in\left[-\frac{B}{2}+f_{c}, \frac{B}{2}+f_{c}\right] .
\end{gathered}
$$

Similarly, Figure 2(a) shows the obtained beampatterns at all the 64 frequencies using the WBFIT approach and the proposed approach. Figure 2(b) compares the beampattern designed by the proposed approach with the WBFIT 
approach at center frequency. The desired sidelobe value and the beamwidth of the desired beampattern are set to $\sigma=$ $-40 \mathrm{~dB}$ and $60^{\circ}$, respectively.

It can be clearly seen that the designed beampattern, using the proposed approach, maintains a constant beamwidth across the entire frequency band from Figure 2(a), and more importantly, the designed sidelobe level is strictly below $-40 \mathrm{~dB}$ from Figure 2(b).

From these simulation examples, compared with the existing WBFIT approach, the proposed approach has achieved significant improvement in both frequency invariant property and sidelobe attenuation. Therefore, the merit of the proposed approach is validated.

\section{Conclusions}

In this paper, a modified approach has been proposed to successfully synthesize the PSDM according to the desired transmit beampattern in wideband MIMO radar systems. With the proposed approach, the mainlobe of the designed beampattern has an almost constant beamwidth for different frequencies and the sidelobe level of the designed beampattern is strictly below a desired sidelobe value (e.g., $-35 \mathrm{~dB}$ and $-40 \mathrm{~dB}$ ). Numerical results were provided to illustrate the effectiveness and validity of the proposed approach.

\section{Conflict of Interests}

The authors declare that there is no conflict of interests regarding the publication of this paper.

\section{Acknowledgment}

This work was supported in part by the Fundamental Research Funds for the Central Universities under Grant no. ZYGX2011YB002.

\section{References}

[1] B. P. Ng, M. H. Er, and C. A. Kot, "Flexible array synthesis method using quadratic programming," IEEE Transactions on Antennas and Propagation, vol. 41, no. 11, pp. 1541-1550, 1993.

[2] S. E. Nai, W. Ser, Z. L. Yu, and H. Chen, "Beampattern synthesis for linear and planar arrays with antenna selection by convex optimization," IEEE Transactions on Antennas and Propagation, vol. 58, no. 12, pp. 3923-3930, 2010.

[3] D. P. Scholnik and J. O. Coleman, "Optimal design of wideband array patterns," in Proceedings of the IEEE International Radar Conference, pp. 172-177, Alexandria, VA, USA, May 2000.

[4] D. R. Fuhrmann and G. S. Antonio, "Transmit beamforming for MIMO radar systems using partial signal correlation," in Proceedings of the 38th Asilomar Conference on Signals, Systems and Computers, pp. 295-299, November 2004.

[5] D. R. Fuhrmann and G. San Antonio, "Transmit beamforming for MIMO radar systems using signal cross-correlation," IEEE Transactions on Aerospace and Electronic Systems, vol. 44, no. 1, pp. 171-186, 2008.

[6] J. Li, P. Stoica, and Y. Xie, "On probing signal design for MIMO radar," in Proceedings of the 40th Asilomar Conference on Signals,
Systems, and Computers, pp. 31-35, Pacific Grove, Calif, USA, 2006.

[7] P. Stoica, J. Li, and Y. Xie, "On probing signal design for MIMO radar," IEEE Transactions on Signal Processing, vol. 55, no. 8, pp. 4151-4161, 2007.

[8] S. Ahmed, J. S. Thompson, Y. R. Petillot, and B. Mulgrew, "Unconstrained synthesis of covariance matrix for MIMO radar transmit beampattern," IEEE Transactions on Signal Processing, vol. 59, no. 8, pp. 3837-3849, 2011.

[9] Y.-C. Wang, X. Wang, H. Liu, and Z.-Q. Luo, "On the design of constant modulus probing signals for MIMO radar," IEEE Transactions on Signal Processing, vol. 60, no. 8, pp. 4432-4438, 2012.

[10] G. S. Antonio and D. R. Fuhrmann, "Beampattern synthesis for wideband MIMO radar systems," in Proceedings of the 1st International Workshop on Computational Advances in MultiSensor Adaptive Processing, pp. 105-108, Puerto Vallarta, Mexico, December 2005.

[11] H. He, P. Stoica, and J. Li, "Wideband MIMO systems: signal design for transmit beampattern synthesis," IEEE Transactions on Signal Processing, vol. 59, no. 2, pp. 618-628, 2011.

[12] T. Yang, T. Su, and Z. Wu, "Fast frequency invariant transmit beampattern synthesis for wideband MIMO radar," in Proceedings of the IET International Conference on Radar Systems (Radar '12), pp. 1-5, Glasgow, UK, October 2012.

[13] P. Gong, Z. Shao, G. Tu, and J. Zhou, "Low PAPR waveform design based on transmit beampattern synthesis for wideband MIMO radars," Science China Information Sciences, vol. 43, no. 3, pp. 429-444, 2013.

[14] H. He, P. Stoica, and J. Li, "Designing unimodular sequence sets with good correlations-including an application to MIMO radar," IEEE Transactions on Signal Processing, vol. 57, no. 11, pp. 4391-4405, 2009.

[15] S. Boyd and L. Vandenberghe, Convex Optimization, Cambridge University Press, 2004.

[16] J. F. Sturm, "Using SeDuMi 1.02, a MATLAB toolbox for optimization over symmetric cones," Optimization Methods and Software, vol. 11, no. 1, pp. 625-653, 1999.

[17] M. Grant and S. Boyd, "CVX: Matlab software for disciplined convex programming (web page and software)," September 2008, http://cvxr.com/cvx/.

[18] Z.-Q. Luo, W.-K. Ma, A. So, Y. Ye, and S. Zhang, "Semidefinite relaxation of quadratic optimization problems," IEEE Signal Processing Magazine, vol. 27, no. 3, pp. 20-34, 2010. 

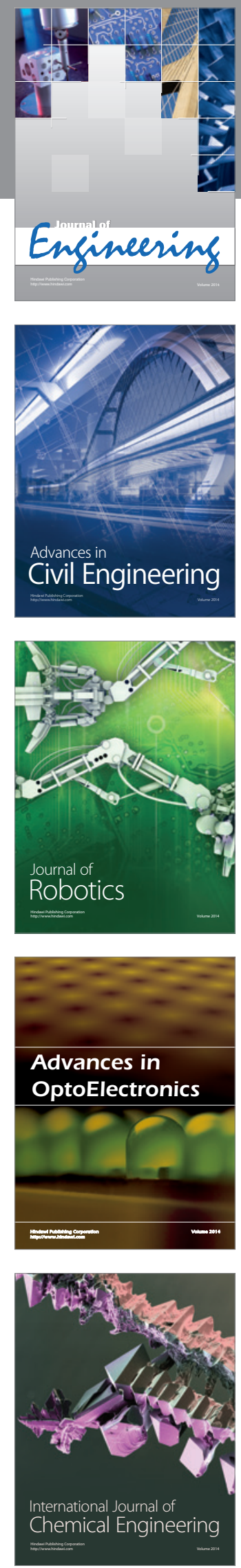

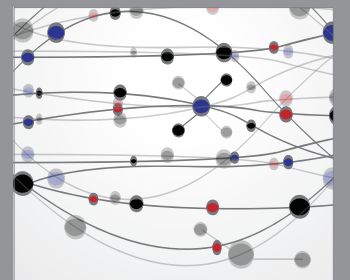

The Scientific World Journal
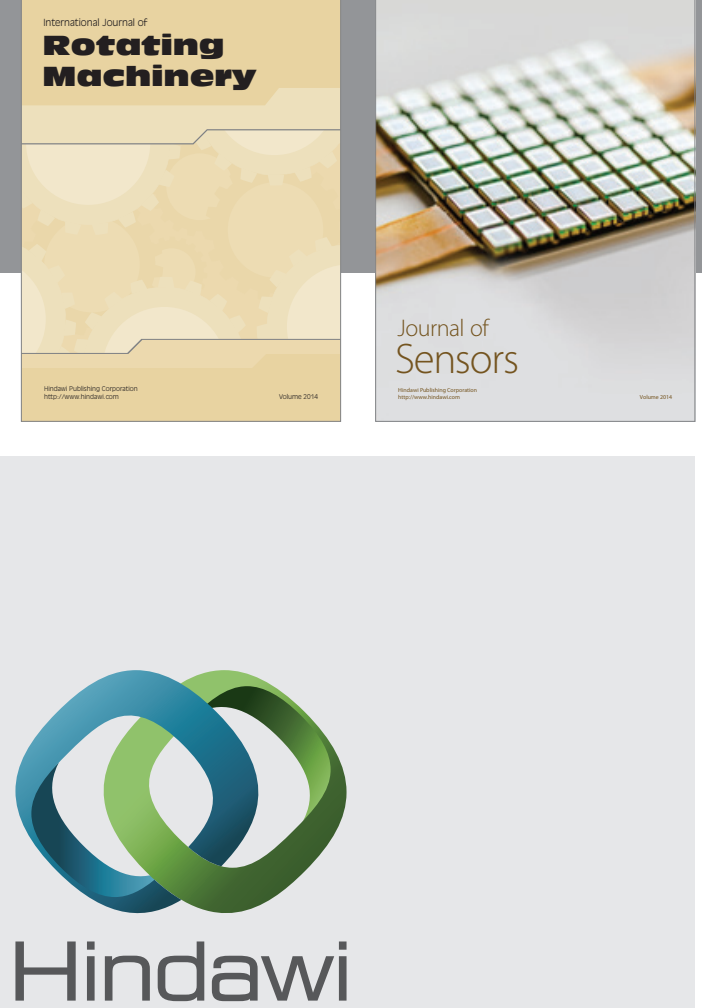

Submit your manuscripts at http://www.hindawi.com
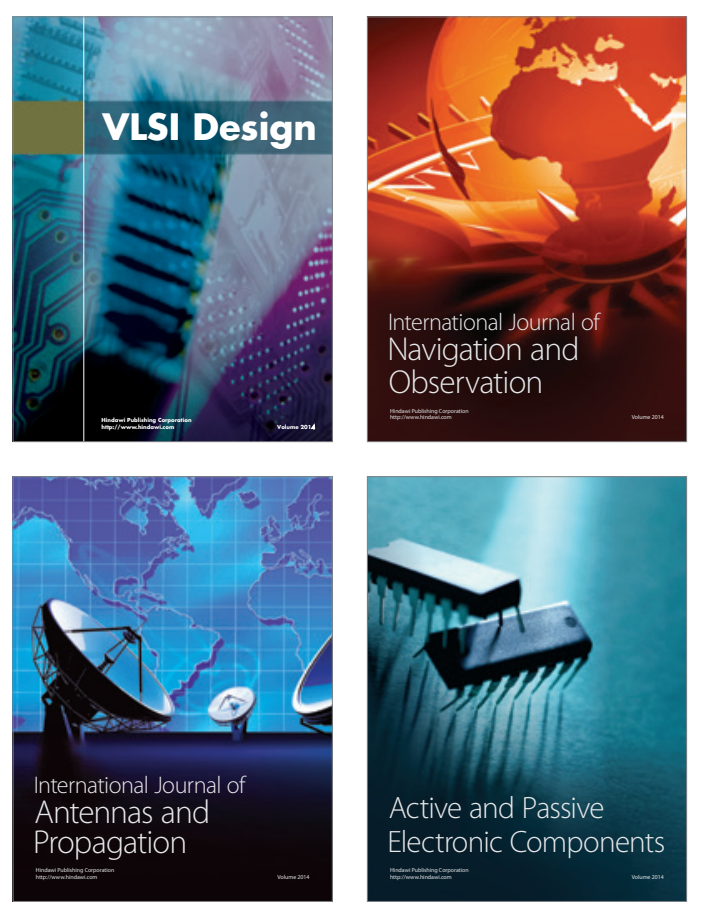
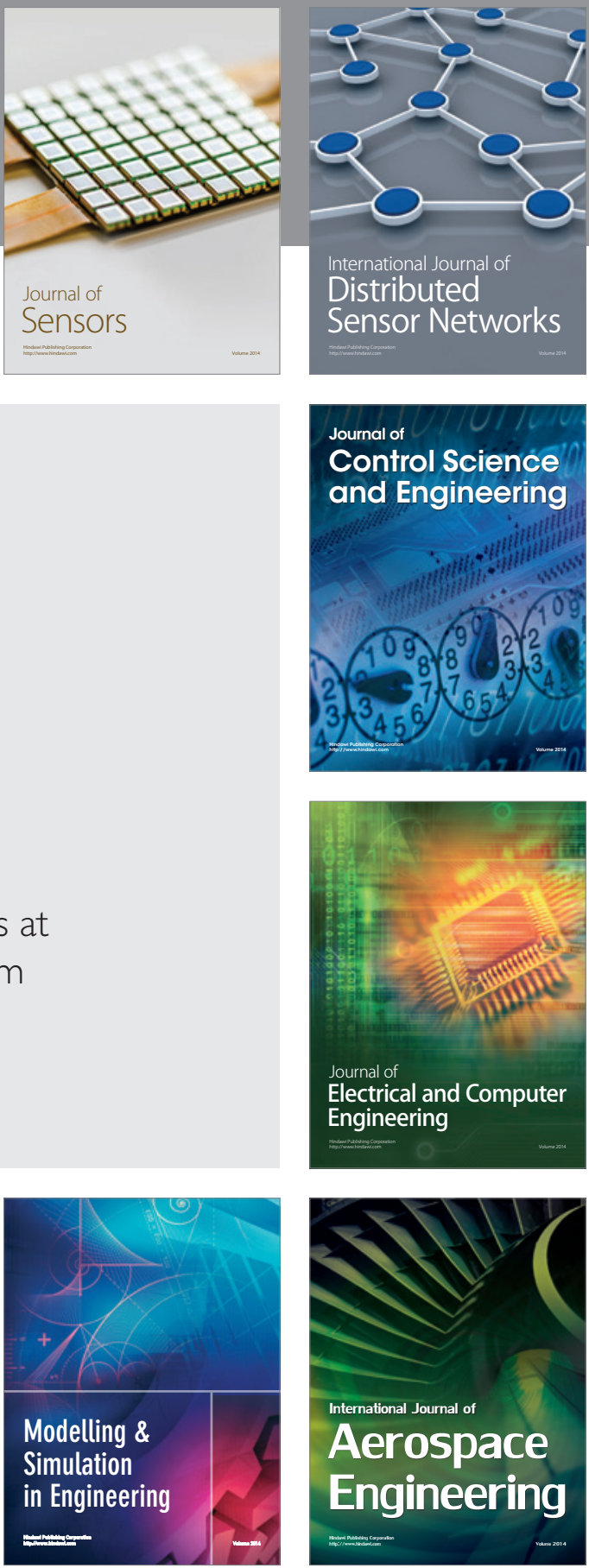

Journal of

Control Science

and Engineering
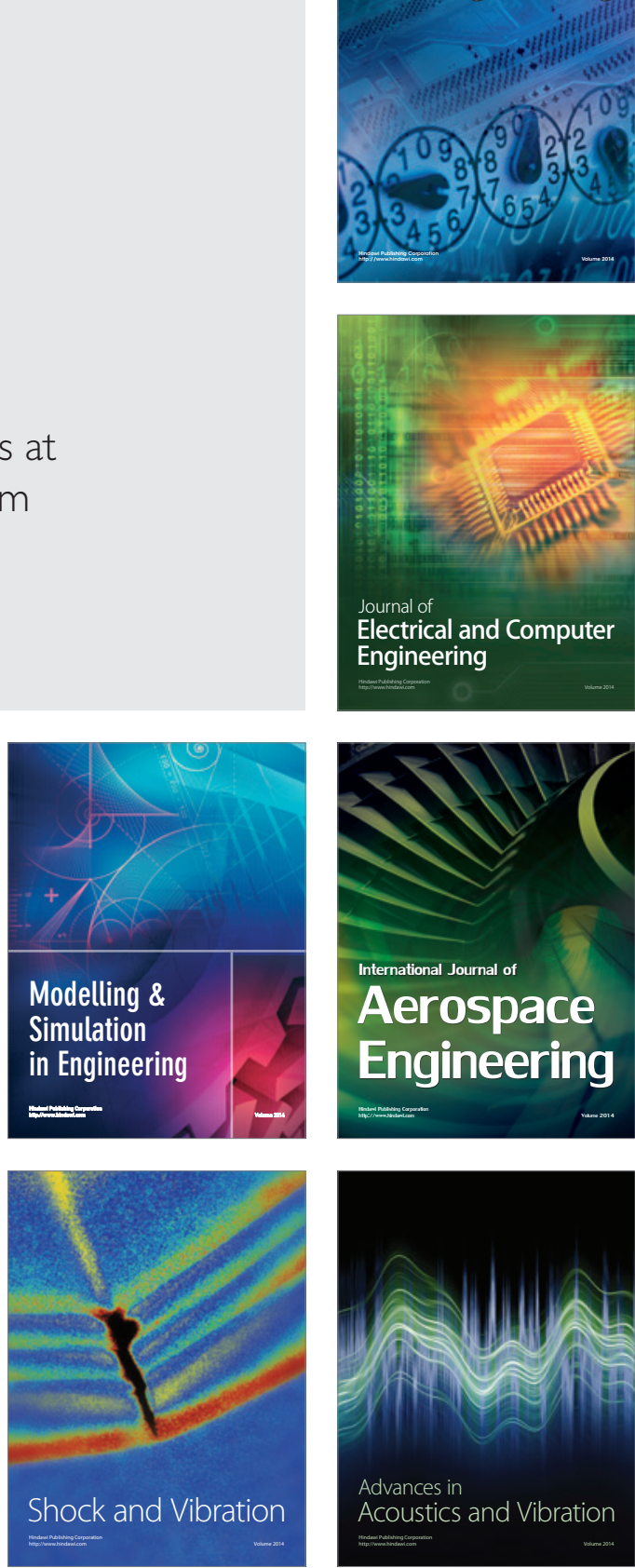